\title{
Short communication: Estimates of genetic parameters for dairy fertility in New Zealand
}

\author{
P. R. Amer, ${ }^{* 1}$ K. Stachowicz, ${ }^{*}$ G. M. Jenkins, ${ }^{*}$ and S. Meier† \\ *AbacusBio Limited, PO Box 5585, Dunedin 9058, New Zealand \\ †DairyNZ, Private Bag 3221, Hamilton 3240, New Zealand
}

\begin{abstract}
Reproductive performance of dairy cows in a seasonal calving system is especially important as cows are required to achieve a 365- $\mathrm{d}$ calving interval. Prior research with a small data set has identified that the genetic evaluation model for fertility could be enhanced by replacing the binary calving rate trait (CR42), which gives the probability of a cow calving within the first $42 \mathrm{~d}$ since the planned start of calving at second, third, and fourth calving, with a continuous version, calving season day (CSD), including a heifer calving season day trait expressed at first calving, removing milk yield, retaining a probability of mating trait (PM21) which gives the probability of a cow being mated within the first $21 \mathrm{~d}$ from the planned start of mating, and first lactation body condition score (BCS), and including gestation length (GL). The aim of this study was to estimate genetic parameters for the proposed new model using a larger data set and compare these with parameters used in the current system. Heritability estimates for CSD and PM21 ranged from 0.013 to 0.019 and from 0.031 to 0.058 , respectively. For the 2 traits that correspond with the ones used in the current genetic evaluation system (mating trait, PM21 and BCS) genetic correlations were lower in this study compared with previous estimates. Genetic correlations between CSD and PM21 across different parities were also lower than the correlations between CR42 and PM21 reported previously. The genetic correlation between heifer CSD and CSD in first parity was 0.66. Estimates of genetic correlations of BCS with CSD were higher than those with PM21. For GL, direct heritability was estimated to be 0.67 , maternal heritability was 0.11 , and maternal repeatability was 0.22 . Direct GL had moderate to high and favorable genetic correlations with evaluated fertility traits, whereas corresponding residual correla-
\end{abstract}

Received September 22, 2015.

Accepted May 10, 2016.

${ }^{1}$ Corresponding author: pamer@abacusbio.co.nz tions remain low, which makes GL a useful candidate predictor trait for fertility in a multiple trait evaluation. The superiority of direct GL genetic component over the maternal GL component for predicting fertility was demonstrated. Future work planned in this area includes the implementation and testing of this new model on national fertility data.

Key words: genetic parameters, fertility, dairy cattle

\section{Short Communication}

The reproductive performance of dairy cows in New Zealand is regarded as superior to many other countries. However, a decline in fertility traits has been observed in recent decades (Burke and Fowler, 2007). In New Zealand, the genetic evaluation of fertility traits is currently performed using methodology described by Harris et al. (2005). To investigate possible new approaches to the genetic evaluation of dairy cow fertility, Bowley et al. (2015) analyzed data from a fertility monitoring project carried out over 2 seasons (2011 and 2012) in 169 herds from different locations across New Zealand (Brownlie et al., 2011). A key recommendation from the analysis was changing calving trait definition from binary to continuous form to increase the accuracy of fertility breeding value predictions [calving season day (CSD), defined as the difference in days between calving and the planned start of calving]. Bowley et al. (2015) also recommended that heifer calving traits be included when redeveloping the genetic evaluation system for cow fertility, which is in agreement with research conducted in other countries (Jamrozik et al., 2005; Pryce et al., 2007; Liu et al., 2008; Tiezzi et al., 2012) showing moderate to high genetic correlations between cow and heifer fertility traits. Notably, heifer calving data are not currently incorporated in the genetic evaluation for fertility in New Zealand.

The findings and recommendations from Bowley et al. (2015) were evaluated by Stachowicz et al. (2014a) using national fertility data. Recommendations from Stachowicz et al. (2014a) to improve accuracy of prediction include replacing the binary calving rate trait (CR42) 
Table 1. Descriptive statistics of the data ${ }^{1}$

\begin{tabular}{lccccc}
\hline Trait & No. of records & Mean & SD & Minimum & Maximum \\
\hline CSD_0 & 14,124 & 17.03 & 17.48 & -50 & 120 \\
CSD_1 & 12,651 & 29.79 & 25.79 & -33 & 129 \\
CSD_2 & 9,248 & 30.50 & 25.24 & -31 & 129 \\
CSD_3 & 6,525 & 24.69 & 18.18 & -30 & 119 \\
PM21_1 & 14,092 & 0.88 & 0.32 & 0 & 1 \\
PM21_2 & 10,844 & 0.87 & 0.34 & 0 & 1 \\
PM21_3 & 6,889 & 0.90 & 0.30 & 0 & 7 \\
BCS & 8,692 & 4.05 & 0.47 & 2 & 7 \\
GL & 28,176 & 281.3 & 4.37 & 242 & 299 \\
\hline
\end{tabular}

${ }^{1} \mathrm{CSD}=$ calving season day in heifers (CSD_0) and in parities 1 to 3 (CSD_1 to CSD_3, respectively); PM21

= percentage mated within $21 \mathrm{~d}$ in parities 1 to 3 (PM21_1 to PM21_3, respectively); GL = gestation length.

with its continuous version CSD, and using BCS and heifer CSD as correlated predictor traits in the fertility genetic evaluation model. These recommendations were found to increase the accuracy of an overall fertility breeding goal trait (Stachowicz et al., 2014b). Including any milk production trait in the model had almost no effect on accuracy of fertility predictions. Hence, Stachowicz et al. (2014b) recommended all milk production traits to be dropped from the fertility genetic evaluation model.

Further work by Stachowicz et al. (2015) using selection index modeling reported a high heritability of gestation length (GL), moderate and favorable genetic correlations between GL and probability of mating in the first $21 \mathrm{~d}$ of a cow's mating period (PM21), and CSD. These results suggest that including GL as a predictor trait would further increase the accuracy of fertility breeding value prediction. Although there are concerns that systematic indirect selection for shorter GL might compromise the viability of calves and replacement heifers with short GL, Jenkins et al. (2016) demonstrated that the detrimental effects of shorter GL are far offset by the benefits (i.e., extra time available to achieve mating and first calving live weight targets).

The fertility, survival, and BCS records used in this study were extracted from the New Zealand national dairy database. They included data from 1989 to 2013. Data were filtered to ensure that only high-quality records were used for variance component estimation (as described in DairyNZ, 2007). Fertility phenotypes for PM21 in lactations 1 to 3 and CSD for heifers and cows in their first 3 lactations were used, as described in detail by Stachowicz et al. (2014a).

Survival records were merged with fertility records to determine if there was a fertility-related culling event for a cow during the first 3 lactations. Cows that were mated but did not calve as a result of this last known mating were penalized by having their calving record created as $21 \mathrm{~d}$ longer than the longest CSD record in their contemporary group (Donoghue et al., 2004).
Cows with a missing mating record had PM21 set to 0 if their CSD was greater than 42. Cows with mating or calving phenotypes (or both) missing and fertilityrelated culling recorded in a given season had PM21 set to 0 , a CSD penalty applied, or both.

First-lactation BCS records were used with one observation per cow preferably at peak lactation (up to $150 \mathrm{~d}$ in milk). The GL as a trait of the calf was defined as the difference in days between last recorded mating and subsequent calving date. The GL records for cows that have fertility phenotypes, as well as for all the calves (both males and females) born to those cows, were included.

To reduce the size of the final data set so that variance component estimation could be performed, herds with contemporary groups of at least 10 cows were randomly sampled. Summary statistics for each trait are presented in Table 1. All cows included in the analysis were required to have a heifer calving record (CSD at first heifer calving). The pedigree with 3 generations of data was used. The total number of animals in the pedigree was 53,614 .

A multiple trait animal model with maternal effects for GL was implemented. It included 9 traits: CSD at first heifer calving, CSD in parities 1 to 3, PM21 in parities 1 to 3, BCS in first lactation, and GL. Both fertility traits (CSD and PM21) were analyzed with the same model, as described in detail by Stachowicz et al. (2014a, 2015). The model used was a slightly modified version of the one currently used in the national genetic evaluation of fertility (Harris et al., 2005). The modification was to include breed-specific heterosis and recombination effects where the Holstein-Friesian breed was partitioned between domestic and overseas Holstein-Friesian. The PM21 was analyzed using a linear model despite being a binary trait. The model used for BCS was based on the one implemented by Harris and Pryce (2004). The GL was evaluated using a model that was consistent with those used previously (Winkelman et al., 2010; Stachowicz et al., 2015). 
Table 2. Estimates of residual correlations (below diagonal), genetic correlations (above diagonal), and heritabilities (on diagonal; bold) ${ }^{1}$

\begin{tabular}{lcccccrrrrr}
\hline Item & CSD_0 & CSD_1 & CSD_2 & CSD_3 & PM21_1 & PM21_2 & PM21_3 & BCS & GL_A & GL_M \\
\hline CSD_0 & $\mathbf{0 . 0 1 9}$ & 0.66 & 0.39 & 0.40 & 0.07 & 0.22 & 0.04 & -0.55 & 0.57 \\
CSD_1 & 0.14 & $\mathbf{0 . 0 1 6}$ & 0.30 & 0.34 & -0.24 & -0.03 & -0.06 & -0.48 & 0.56 \\
CSD_2 & 0.01 & 0.36 & $\mathbf{0 . 0 1 3}$ & 0.70 & -0.49 & -0.58 & -0.42 & -0.46 & 0.45 \\
CSD_3 & 0.02 & 0.04 & 0.13 & $\mathbf{0 . 0 1 9}$ & -0.50 & -0.47 & -0.58 & -0.50 & 0.38 \\
PM21_1 & -0.11 & -0.17 & -0.04 & -0.01 & $\mathbf{0 . 0 3 1}$ & 0.54 & 0.52 & 0.19 & -0.15 & -0.09 \\
PM21_2 & -0.04 & -0.06 & -0.15 & -0.05 & 0.10 & $\mathbf{0 . 0 5 8}$ & 0.77 & 0.30 & -0.21 \\
PM21_3 & -0.04 & -0.06 & -0.08 & -0.27 & 0.08 & 0.14 & $\mathbf{0 . 0 5 2}$ & 0.28 & -0.22 \\
BCS & 0.03 & -0.02 & -0.05 & -0.02 & 0.07 & 0.01 & 0.02 & $\mathbf{0 . 1 1 7}$ & 0.02 \\
GL & 0.03 & -0.02 & 0.07 & 0.08 & 0.09 & 0.03 & 0.04 & 0.02 & $\mathbf{0 . 6 7 3}$ & -0.01 \\
\hline
\end{tabular}

${ }^{1} \mathrm{CSD}=$ calving season day in heifers (CSD_0) and in parities 1 to 3 (CSD_1 to CSD_3, respectively); PM21 = percentage mated within 21 d in parities 1 to 3 (PM21_1 to PM21_3, respectively); GL = gestation length; $\mathrm{A}=$ direct animal effect; $\mathrm{M}=$ maternal effect.

${ }^{2}$ For GL, the maternal genetic heritability was 0.108 and repeatability was 0.216 .

Genetic analysis was carried out using ASReml3 software, which fits linear mixed models using REML methods (Gilmour et al., 2009).

Estimates of genetic and phenotypic parameters are presented in Table 2. Standard errors of the estimates are not presented as they were unrealistically high for the presented model. During construction of multi-trait model, subsets of traits were analyzed and as more traits were added to the model, it was observed that the estimates of genetic parameters remained very similar, whereas standard error increased substantially (data not presented). Adding parameters in ASReml that are correlated with the old parameters causes the conditional information on a parameter to decrease and so the inverse of the information matrix increases. The higher the correlation, the greater the increase in variance, which leads to inflation of the standard error.

Heritability estimates for CSD ranged from 0.013 to 0.019 , which is slightly lower than for its binomial equivalent (CR42) as currently used (Harris et al., 2005). From initial results reported by Stachowicz et al. (2014a), it was expected that CSD would have higher heritabilities than CR42. However, as demonstrated by Donoghue et al. (2004), when the penalty was applied to phenotypes of cows that were mated but did not calve, it resulted in higher estimates of both residual and additive genetic variance, and lower estimates of heritabilities than for uncensored data. Applying the penalties was still recommended by Donoghue et al. (2004), as the lower estimate of additive genetic variance when censored data were ignored (in conjunction with more biased estimated breeding values of sires) highlighted the suitability of applying a penalty for genetic evaluation.

For GL, the direct heritability, maternal heritability, and maternal repeatability were $0.67,0.11$, and 0.22 , respectively (Table 2). Previously reported heritability values for GL in New Zealand were 0.44 (Winkelman and Spelman, 2001) and 0.52 (Winkelman et al., 2010).
Both studies used a sire model without maternal genetic effects fitted. Using comparable models, Johanson et al. (2011) reported the direct heritability of GL to be 0.51 , maternal heritability of 0.08 , and repeatability of 0.54, whereas Eaglen et al. (2013) estimated direct and maternal heritability of GL to be 0.49 and 0.09 , respectively.

Current estimates for the genetic correlations among PM21 traits in different parities ranged from 0.52 to 0.77 and were lower than previous estimates (0.85 to 0.92; Harris et al., 2005). The genetic correlations for BCS with PM21 observed here were also lower than those estimated by Harris et al. (2005; 0.19 to 0.30 vs. 0.43 to 0.49 , respectively). Genetic correlations between CSD and PM21 across different parities were also lower than those reported by Harris et al. (2005) between CR42 and PM21, after accounting for the reversal of sign due to the change in trait definition. The genetic correlation between heifer CSD and CSD in first parity was 0.66 , which is in agreement with the value of 0.70 reported by Bowley et al. (2015).

Estimates of genetic correlations of BCS with CSD and PM21 varied for different parities, and were higher for CSD than for PM21. Results for genetic and residual correlations of BCS with CSD traits shown in Table 2 were taken from a subset analysis involving these traits only. Corresponding results from a larger analysis gave results that were inconsistent with correlations of estimated breeding values calculated on a full industry dataset but when no genetic correlations were included in the genetic evaluation step used to obtain the estimated breeding values.

The GL direct was found to be a trait with very high direct heritability (0.67) as well as moderate to high and favorable genetic correlations with evaluated fertility traits ( -0.15 to -0.22 with PM21 and 0.38 to 0.57 for CSD). Corresponding residual correlations were low, which makes GL a strong candidate as a predictor trait for fertility in a multiple trait model. Indirect selection 
pressure on GL is already occurring in New Zealand, which is reflected as an observed downward trend in GL breeding values (unpublished data). Genetic correlations between GL and other fertility traits such as calving interval and nonreturn rates were reported to be approximately 0.2 in other studies (Jamrozik et al., 2005; Eaglen et al., 2013).

Covariance components presented in this paper will be used in a new genetic evaluation model for fertility traits of the entire New Zealand dairy population. The new model has the potential to further improve accuracy of prediction of fertility breeding values.

\section{ACKNOWLEDGMENTS}

This project is funded by New Zealand dairy farmers through DairyNZ Incorporated (RD1402; Hamilton, New Zealand) and the Ministry of Primary Industries (Wellington, New Zealand) through the Primary Growth Partnership.

\section{REFERENCES}

Bowley, F. E., R. E. Green, P. R. Amer, and S. Meier. 2015. Novel approaches to genetic analysis of fertility traits in New Zealand dairy cattle. J. Dairy Sci. 98:2005-2012.

Brownlie, T. S., A. M. Weir, I. Tarbotton, J. M. Morton, C. Heuer, and S. McDougall. 2011. Reproductive management of dairy herds in New Zealand: Attitudes, priorities and constraints perceived by farmers managing seasonal-calving, pasture-based herds in four regions. N. Z. Vet. J. 59:28-39.

Burke, C. R., and C. Fowler. 2007. Fertility in New Zealand dairy herds: Industry situation and a way forward for improving on-farm reproductive performance. Pages 194-202 in Proc. South Island Dairy Event (SIDE). Accessed Jun. 3, 2016. http://side.org.nz/ wp-content/uploads/2014/05/Fertility-in-New-Zealand-dairyherds.pdf.

DairyNZ. 2007. Contemporary groups for New Zealand dairy herd testing. Accessed Jan. 20, 2013. http://www.dairynz.co.nz/ media/580117/Contemporary-Grouping-May-2007.pdf.

Donoghue, K. A., R. Rekaya, and J. K. Bertrand. 2004. Comparison of methods for handling censored records in beef fertility data: Field data. J. Anim. Sci. 82:357-361.
Eaglen, S. A. E., M. P. Coffey, J. A. Woolliams, and E. Wall. 2013 Direct and maternal genetic relationships between calving ease, gestation length, milk production, fertility, type, and lifespan of Holstein-Friesian primiparous cows. J. Dairy Sci. 96:4015-4025.

Gilmour, A. R., B. J. Gogel, B. R. Cullis, and R. Thompson. 2009. ASReml User Guide Release 3.0. VSN International Ltd., Hemel Hempstead, UK. www.vsni.co.uk.

Harris, B. L., and J. E. Pryce. 2004. Genetic and phenotypic relationships between milk protein percentage, reproductive performance and body condition score in New Zealand dairy cattle. Proc. N.Z. Soc. Anim. Prod. 64:127-131.

Harris, B. L., J. E. Pryce, Z. Z. Xu, and W. A. Montgomerie. 2005. Fertility breeding values in New Zealand, next generation. Interbull Bull. 33:47-50.

Jamrozik, J., J. Fatehi, G. Kistemaker, and L. R. Schaeffer. 2005. Estimates of genetic parameters for Canadian Holstein female reproduction traits. J. Dairy Sci. 88:2199-2208.

Jenkins, G. M., P. R. Amer, K. Stachowicz, and S. Meier. 2016. Phenotypic associations between gestation length, fertility, survival, and calf traits. J. Dairy Sci. 99:418-426.

Johanson, J. M., P. J. Berger, S. Tsuruta, and I. M. Isztal. 2011. A Bayesian threshold-linear model evaluation of perinatal mortality, dystocia, birth weight, and gestation length in a Holstein herd. J. Dairy Sci. 94:450-460.

Liu, Z., J. Jaitner, F. Reinhardt, E. Pasman, S. Rensing, and R. Reents. 2008. Genetic evaluation of fertility traits of dairy cattle using multiple-trait animal model. J. Dairy Sci. 91:4333-4343.

Pryce, J. E., B. L. Harris, and L. R. McNaughton. 2007. The genetic relationship between heifer and cow fertility. Proc. N.Z. Soc. Anim. Prod. 67:388-391.

Stachowicz, K., G. M. Jenkins, P. R. Amer, J. R. Bryant, and S. Meier. 2015. Proposed changes in the genetic evaluation of dairy fertility in New Zealand. Proc. Assoc. Advmt. Anim. Breed. Genet. $21: 370-373$.

Stachowicz, K., G. M. Jenkins, P. R. Amer, and S. Meier. 2014a. New approaches to fertility evaluation in a seasonal calving system. Interbull Bull. 48:89-93.

Stachowicz, K., G. M. Jenkins, N. A. Dennis, P. R. Amer, J. R. Bryant, and S. Meier. 2014b. Evaluation of the dairy fertility evaluation model using selection index theory. Proc. Australasian Dairy Sci. Symp. 5:65-67.

Tiezzi, F., C. Maltecca, A. Cecchinato, M. Penasa, and G. Bittante. 2012. Genetic parameters for fertility of dairy heifers and cows at different parities and relationships with production traits in first lactation. J. Dairy Sci. 95:7355-7362.

Winkelman, A. M., and R. J. Spelman. 2001. Selection for reduced gestation length in New Zealand dairy cattle. Proc. Assoc. Advmt. Anim. Breed. Genet. 14:63-66.

Winkelman, A. M., R. M. Wood, and B. L. Harris. 2010. Enhancement of calving difficulty breeding values in New Zealand. Interbull Bull. 42:91-94. 